\title{
DUST AND IONIZED GAS IN ACTIVE RADIO ELLIPTICAL GALAXIES
}

\author{
D.A. Forbes ${ }^{1,2}$, W.B. Sparks ${ }^{1}$ and F.D. Macchetto ${ }^{1,3}$ \\ ${ }^{1}$ Space Telescope Science Institute, 3700 San Martin Drive, Baltimore MD 21218* \\ ${ }^{2}$ Institute of Astronomy, Madingley Road, Cambridge CB3 0HA, U.K.
}

\begin{abstract}
We present broad and narrow bandwidth imaging of three southern elliptical galaxies which have flat-spectrum active radio cores (NGC 1052, IC 1459 and NGC 6958). All three contain dust and extended low excitation optical line emission, particularly extensive in the case of NGC 1052 which has a large $\mathrm{H}_{\alpha}+$ [NII] luminosity. Both NGC 1052 and IC 1459 have a spiral morphology in emission-line images. All three display independent strong evidence that a merger or infall event has recently occurred, i.e., extensive and infalling HI gas in NGC 1052, a counter-rotating core in IC 1459 and Malin-Carter shells in NGC 6958. This infall event is the most likely origin for the emission-line gas and dust, and we a currently investigating possible excitation mechanisms (Sparks et al. 1990).
\end{abstract}

\section{INTRODUCTION}

Optical line emission, radio emission, nuclear activity and the presence of $\mathrm{X}$-ray emitting coronal halos often occur together in elliptical galaxies (e.g., Baum et al. 1988). There is good evidence to link optical line emission with mergers, or infall events, when in the presence of counter-rotating cores, dust lanes, discrete stellar components and Malin-Carter shells for example. Sparks et al. (1989) proposed that the cooler infalling gas can significantly modify the properties of the ambient hot medium via thermal conduction, and that the large mass deposition rates inferred from $X$-ray observations are not present (see also Sparks 1989, this meeting).

Here we present CCD continuum and emission-line imaging obtained using EFOSC on the ESO 3.6-m telescope at $\mathrm{La}$ Silla, Chile. We investigate three nearby active elliptical galaxies which show independent evidence that infall has recently occurred; namely NGC 1052, IC 1459 and NGC 6958. All three objects were known to have flat-spectrum radio-core emission (Sparks et al. 1984) and dust features (Sparks et al. 1985). Thomas et al. (1986) present and interpret $\mathrm{X}$-ray observations of two of these galaxies in terms of a 'standard' cooling-flow model. Our observations taken together with data already available lead us to conclude that the most likely origin for the extensive emission-line filament systems and dust patches which we find, is an external one.

The observations consist of short $(\sim 3 \mathrm{sec})$, medium and long $(\sim 1200 \mathrm{sec})$ exposures with typical seeing of 1.5 arcsec. Images in the same band were merged, with $1 / \sigma^{2}$ weighting to maximise signal-to-noise, to give a master image free of saturated regions. Ellipses were fitted to the master images and models of the underlying light distribution were thereby constructed. Finally the data was calibrated using aperture photometry and absolute flux determinations made. A value of $\mathrm{H}_{\mathrm{o}}=50 \mathrm{~km} \mathrm{~s}^{-1} \mathrm{Mpc}^{-1}$ is used throughout. 
NGC 1052

Properties:

- Distance $=29.31 \mathrm{Mpc}$.

- Contains $a \approx 1 \mathrm{Jy}$ flat-spectrum variable radio core, a VLBI jet, and relatively compact double radio source (Fosbury et al. 1978; Wrobel 1984; Davies and Illingworth 1986).

- $\mathrm{HI}$ mass $=11.5 \times 10^{8} \mathrm{M}_{\odot}$ (van Gorkom et al. 1986).

- $\mathrm{L}_{X}=5.46 \times 10^{40} \mathrm{erg} \mathrm{s}^{-1}$ (Fabbiano et al. 1989); a typical value for an elliptical with a large non-stellar contribution from a hot gaseous halo.

- IRAS measurements suggest a long wavelength infrared excess consistent with thermal radiation from $\sim 10^{7} \mathrm{M}_{\odot}$ of dust (Knapp et al. 1989).

- Exhibits extended LINER emission (Fosbury et al. 1978; Davies and Illingworth 1986).

Evidence for a Merger:

- The large misalignment of the rotation axis of the stars compared to the HI and ionized gas, and its high angular momentum strongly suggests that it has been accreted rather than arising from mass loss from the stars (Davies and Illingworth 1986).

- The HI gas shows a 'tidal tail', indicative of a recent merger (van Gorkom et al. 1986).

- HI is seen in absorption at both the galaxy systemic velocity and redshifted, implying the presence of infalling gas (van Gorkom et al. 1986).

- CCD images revealed the presence of a prominent minor axis dust-lanes (see Sparks et al. 1985 and Fig. 1). confirming that the gas is not of primordial origin.

- A gas-to-dust ratio of $\sim 100$, normal for spiral galaxies, also suggests an accretion origin rather than primordial or internal stellar mass loss processes (van Gorkom et al. 1986).

Results:

The image of dust optical depth (Fig. 1) shows far more extensive absorption than previously seen (Sparks et al. 1985) i.e., additional dust covers essentially the whole Western half of the galaxy, with structure primarily in the minor axis direction. The emission-line image (Fig. 2) displays an irregular spiral morphology. These together suggest an obvious model for the system, whereby we are observing an inclined disc of cool gas passing through the galaxy and intersecting the nucleus. The sense of the spiral pattern is correct for a trailing spiral if the inclination is as suggested by the dust i.e., 'in front' to the West (kinematics from Davies and Illingworth 1986). We therefore favor a situation whereby the HI gas lies in a disc and is ionized in its inner regions either by the active nucleus or by interaction with the hot $\mathrm{X}$-ray gas associated with the galaxy. It is seen in absorption where it crosses in front of the galaxy (as proposed by Sparks et al. 1985), while the optical emission-line gas is seen on both sides. Note that this implies (i) the gas cannot be primordial (ii) a spherical distribution for the filament system is unlikely.

We find the $\mathrm{H}_{\alpha}+[\mathrm{NII}]$ emission extends about 40 arcsec from the center of the galaxy with a total luminosity of $1.4 \times 10^{41} \mathrm{erg}^{-1}$. 


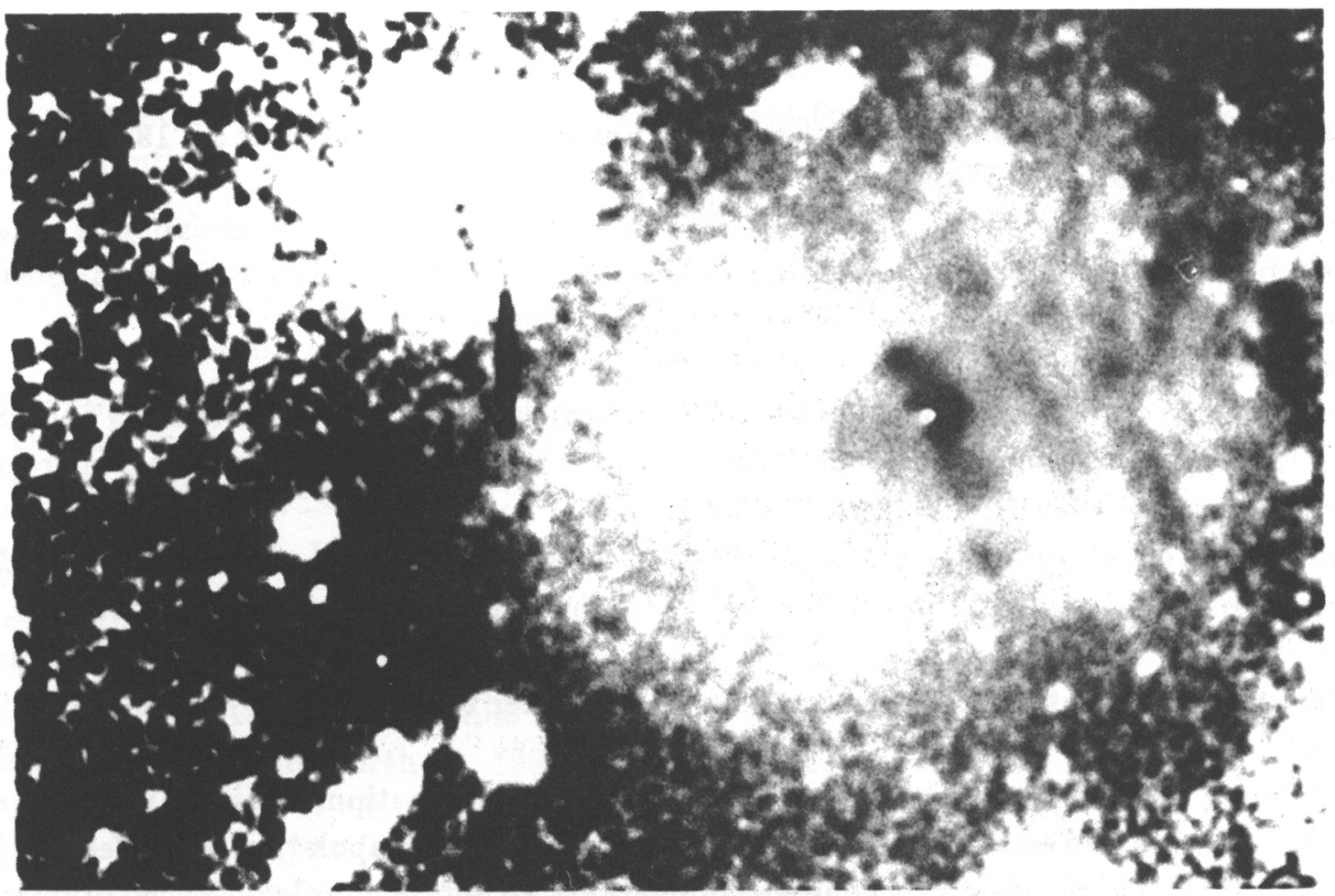

Figure 1 B-R dust map for NGC 1052. Note extensive dust lanes parallel to the minor axis on the West side of the galaxy. North is up and East is left in all figures, which have a width $\approx 3.7$ arrmin

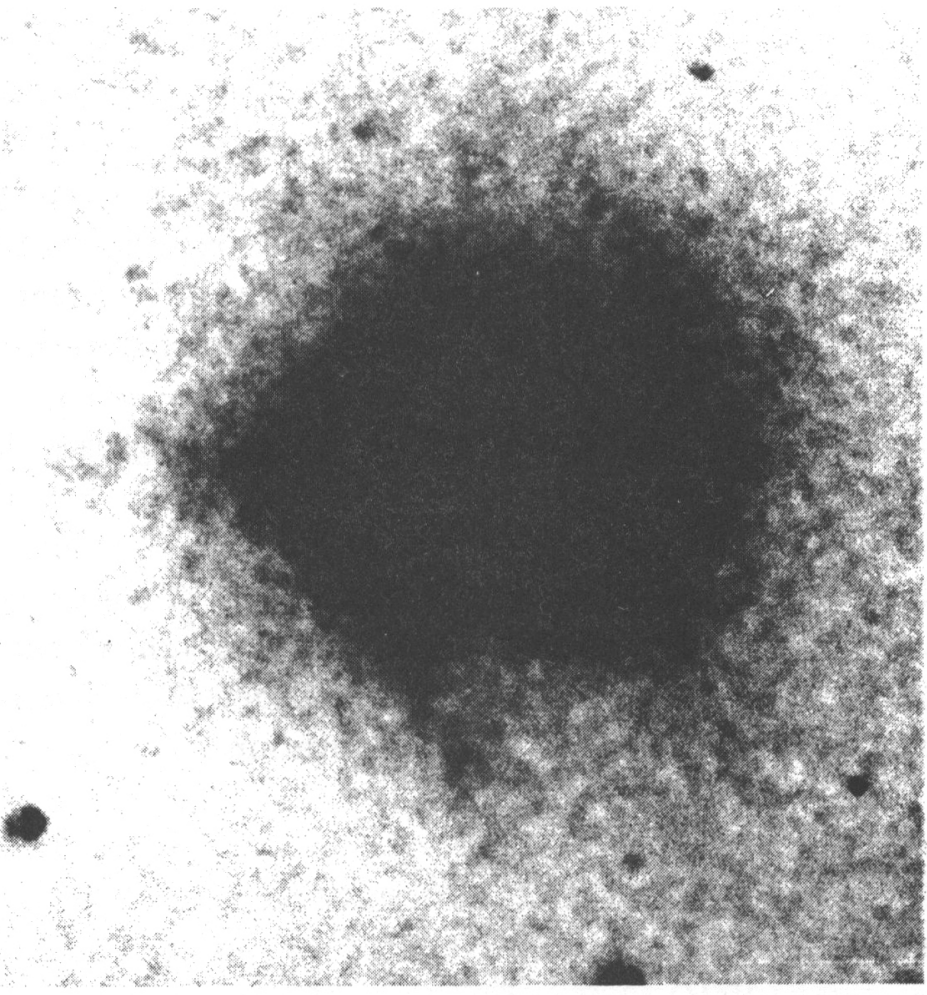

Figure $2 \mathrm{H}_{\alpha}+[\mathrm{NII}]$ image for NGC 1052 after continuum subtraction. 


\section{IC 1459}

Properties:

- Distance $=32.77$ Mpc.

- Has a dominant $\approx 1$ Jy non-variable flat-spectrum radio core (Sparks et al. 1984).

- $\mathrm{HI}$ mass $<7.5 \times 10^{8} \mathrm{M}_{\odot}$ (Walsh et al. 1989).

- $\mathrm{L}_{X}=4.30 \times 10^{40} \mathrm{erg} \mathrm{s} \mathrm{s}^{-1}$ (Fabbiano et al. 1989); we find the X-ray emission is extended probably due to a hot gaseous halo ( some contribution from discrete stellar sources is possible). - IRAS measurements suggest $\sim 10^{7} \mathrm{M}_{\odot}$ of dust (Knapp et al. 1989).

Evidence for a merger:

- Exhibits a counter-rotating core (Franx and Illingworth 1988), in which the stars and gas are rotating in opposite directions, strongly suggesting that a 'cold' stellar system has merged and settled at the core (Balcells and Quinn 1989).

- Photographic enhancement reveals a faint one-arm extended spiral structure (Malin 1985), although some form of interaction with the companion galaxies may well be responsible.

- It has strongly 'twisted isophotes' (Evans 1951; Williams and Schwarzchild 1979). This may be indicative of a recent merger or interaction (see Bender et al. 1989).

\section{Results:}

We find a long single 'spiral arm' emission feature, with a suggestion of fainter irregular structure. The direction and sense of the arm is consistent with an extrapolation of the feature found by Malin (1985). To maintain this coherent filamentary structure over length scales larger than the optical image of the galaxy again implies an external infall origin.

\section{NGC 6958}

\section{Properties:}

- Distance $=53.41 \mathrm{Mpc}$.

- Exhibits a weak flat-spectrum radio core and is peculiarly blue for a radio elliptical (Sparks et al. 1984), although it has a very red nucleus (Sparks et al. 1986).

- HI mass $<15.3 \times 10^{8} \mathrm{M}_{\odot}$ (Walsh et al. 1989).

- The IRAS data suggests a cool ISM is present (Walsh et al. 1989). Wilkinson et al. (1987) find $\log \left(\mathrm{F}_{I_{R}} / \mathrm{F}_{B}\right)=0.89$, indicating a very high star formation rate for an elliptical.

Evidence for a Merger:

- The presence of Malin-Carter shells strongly suggests the recent infall of a small galaxy about $10^{\circ}$ years ago (Quinn 1984).

\section{Results:}

We find dust patches $\sim 30$ arcsec to the East of the nucleus much more clearly than in Sparks et al. (1985), and in addition that the dust extends over the whole of the Northern half of the image. A long slit spectrum shows extended low excitation line emission with a considerable velocity gradient indicative of rapid rotation.

\section{SUMMARY}

We find NGC 1052 and NGC 6958 have much more extensive dust than previously realised. All three galaxies contain highly extended regions of optical line emission, with a spiral morphology in the cases of NGC 1052 and IC 1459. An external origin for these cooler gaseous components is favoured. Our goal in these investigations is to understand the excitation mechanism of optical 
line emission in elliptical galaxies and to understand the relationships between the different phases of the ISM. We wish to know if the infall process can affect the $\mathrm{X}$-ray halo properties of elliptical galaxies, perhaps removing the requirement for the relatively large mass depositions of the conventional cooling-flow picture.

Two very different models for NGC 1052 are currently being considered (Sparks et al. 1990). One is photoionization by an active nucleus, the other thermal electron conduction in which the emission flux is assumed to be proportional to the energy available by conduction. There are few free parameters in our thermal conduction model, yet with plausible values a remarkably good fit is obtained to the surface brightness profile. In conclusion, we deduce that mergers can, from an optical perspective, appear quite similar to cooling flows. To establish how fundamental these similarities are further work is required.

\section{References}

Balcells, M., and Quinn, P. J. 1989, Astrophys. and Space Sci., 156, 133.

Baum, S. A., et al. 1988, Ap. J. (Suppl, 68, 833.

Bender, R., et al. 1989, Astron. and Astr., 217, 35.

Davies, R. L., and Illingworth, G. 1986, Ap. J., 302, 234.

Evans, D. S. 1951, M. N. R. A. S., 111, 526.

Fabbiano, G., Gioia, I. M., and Trinchieri, G. 1989, Ap. J., 347, 127.

Fosbury, R. A. E., et al. 1978, M. N. R. A. S., 183, 549.

Franx, M., and Illingworth, G. 1988, Ap. J. (Letters), 327, L55.

Malin, D. F. 1985, in New Aspects of Galaxy Photometry, ed. J. L. Nieto (Berlin: SpringerVerlag) p27.

Quinn, P. J. 1984, Ap. J., 279, 596.

Knapp, G. R., Kerr, F. J., and Williams, B. A. 1978, Ap. J., 222, 800.

Knapp, G. R., Beis, W. E., and van Gorkom, J. H. 1989, preprint.

Sparks, W. B., et al. 1984, M. N. R. A. S., 207, 445.

Sparks, W. B., et al. 1985, M. N. R. A. S., 217, 87.

Sparks, W. B., Hough, J. H., Axon, D. J., and Bailey, J. 1986, M. N. R. A. S., 218, 429.

Sparks, W. B., Macchetto, F. D., and Golombek, D. 1989, Ap. J., 345, 153.

Sparks, W. B., Forbes, D. A., Macchetto, F. D. and Golombek, D. 1990, in preparation.

Thomas, P. A., et al. 1986, M. N. R. A. S., 222, 655.

van Gorkom, J. H., et al. 1986, A. J., $81,791$.

Walsh, D. E. P., et al. 1989, submitted to Ap. J.

Williams, T. B., and Schwarzchild, M. 1979, Ap. J., 227, 56.

Wilkinson, A., Browne, I. W. A., and Wolstencroft, R. D. 1987, M. N. R. A. S., 228, 933.

Wrobel, J. M. 1984, Ap. J., 284, 531.

${ }^{3}$ Affiliated with the Astrophysics Division, Space Science Department, European Space Agency (ESA).

- Operated by the Association of Universities for Research in Astronomy. Inc., under contract with the National Aeronautics and Space Administration. 